\title{
Pre-interventional assessment and calcification score of the aortic valve and annulus, with multi-detector CT, in transcatheter aortic valve implantation (TAVI) using the Medtronic CoreValve
}

\author{
Authors: \\ Antoinette Reinders \\ Coert S. de Vries ${ }^{1}$ \\ G. Joubert ${ }^{2}$

\begin{abstract}
Affiliation:
${ }^{1}$ Department of Clinical of the Free State, South Africa
\end{abstract} \\ Imaging Sciences, University \\ ${ }^{2}$ Department of Biostatistics, \\ University of the Free State, \\ South Africa \\ Correspondence to: \\ Antoinette Reinders \\ Email: \\ antoinette.reinders@gmail. \\ com \\ Postal address: \\ 1 Logeman Street, \\ Universitas 9301, \\ Bloemfontein, South Africa \\ Dates: \\ Received: 03 Dec. 2014 \\ Accepted: 12 Feb. 2015 \\ Published: 04 June 2015 \\ How to cite this article: \\ Reinders A, De Vries \\ CS, Joubert, G. Pre- \\ interventional assessment \\ and calcification score of the \\ aortic valve and annulus, \\ with multi-detector $\mathrm{CT}$, in \\ transcatheter aortic valve \\ implantation (TAVI) using \\ the Medtronic CoreValve. S \\ Afr J Rad. 2015;19(1); Art. \\ \#762, 7 pages. http://dx.doi. \\ org/10.4102/sajr.v19i1.762

\section{Copyright:} \\ C 2015. The Authors. \\ Licensee: AOSIS \\ OpenJournals. This work is \\ licensed under the Creative \\ Commons Attribution \\ License.

\section{Read online:}

Background: Transcatheter aortic valve implantation (TAVI) provides an acceptable alternative for aortic valve replacement in the elderly, but needs accurate pre-procedural imaging to optimise intervention.

Objectives: To evaluate an alternative manual aortic valve calcification scoring system with computed tomography, for patients undergoing TAVI. We hypothesise a correlation between the Free State aortic valve calcium computed tomography score (FACTS) scoring system, valve plaque density and procedure-related complications.

Methods: Twenty patients suitable for TAVI were selected according to standard international guidelines and received multimodality imaging prior to intervention. Images were reviewed by two reviewers who were blinded to each other's scores. Where large inter-individual score variations existed, retraining was done and scores repeated, using a double-blinded method. Matched scores were included in the final analysis. Rosenhek calcification scores were used as a standard of reference.

Results: The study comprised 9 (45\%) men and 11 (55\%) women, with a median age of 83.5 years. Median EuroSCORE was 15.5. FACTS scores $\geq 6$ were associated with the presence of a paravalvular leak $(p=0.01)$. Procedure-related complications (left bundle branch block, repositioning of the valve and anaemia) were seen in patients with plaques measuring $\geq 1000$ HU $(p=0.07)$.

Conclusion: The FACTS score and averaged valve plaque HU showed potential for predicting a paravalvular leak and procedure-related complications, and could be valuable in the future for optimising patient selection for TAVI.

\section{Introduction}

Aortic stenosis is a global major debilitating health concern that is associated with high mortality and morbidity in the elderly population. Until recently, the only treatment for such patients was surgical aortic valve replacement, showing reasonable peri-operative results, but excluding a large number of symptomatic patients owing to unacceptably high peri-operative risk as indicated by a European Society of Cardiology score $>20 \%$ (EuroSCORE) or Society of Thoracic Surgeons (STS) score $>10.1$

In the above pre-interventional scoring systems, patients are allocated specific numerical values according to comorbid conditions such as hypertension, hypercholesterolaemia and chronic obstructive pulmonary disease. Since the early 1990s, alternative routes for aortic valve replacement have been sought. ${ }^{1,2,3}$ Transcatheter aortic valve replacement (TAVI) has emerged as a suitable and clinically better-tolerated alternative to surgical aortic valve replacement in selected patients. ${ }^{1}$ Results from the PARTNER trial (cohort B) showed an absolute reduction in one-year mortality of $20 \%$ with marked and durable improvement in functional status and reduced hospitalisation in patients who had undergone TAVI, compared with those who had been treated medically. ${ }^{1}$

Because TAVI is carried out in selected high-risk patients, pre-interventional assessment of the cardiac and aorta iliac anatomy is of the utmost importance. ${ }^{4}$ Trials have shown multi-detector computed tomography (MDCT) emerging as a valid alternative, and is in some institutions preferred to catheter angiography in assessing the aorta and ilio-femoral circulation prior to valve deployment. This trend is of particular importance in planning a percutaneous entry site as 
TABLE 1: Sellers proposed echocardiogram grading for aortic regurgitation.

\begin{tabular}{|c|c|c|c|}
\hline AR severity & Mild & Moderate & Severe \\
\hline \multirow[t]{3}{*}{ Specific signs } & Vena contracta $<0.3 \mathrm{~cm}$ & Intermediate values & Vena contracta $\geq 0.6 \mathrm{~cm}$ \\
\hline & Central jet width $<25 \%$ of LVOT & Intermediate values & Central jet width $>65 \%$ of LVOT \\
\hline & $\begin{array}{l}\text { No or brief early diastolic flow reversal } \\
\text { in descending aorta }\end{array}$ & - & $\begin{array}{l}\text { Holodiastolic flow reversal in descending } \\
\text { aorta. Flail or wide coaptation defect }\end{array}$ \\
\hline \multirow[t]{2}{*}{ Supportive signs } & $\mathrm{PHT}>500 \mathrm{~ms}$ & - & PHT $<200 \mathrm{~ms}$ \\
\hline & No or minimal flow convergence & - & $\begin{array}{l}\text { Large flow convergence. Moderate or } \\
\text { greater LV enlargement }\end{array}$ \\
\hline Reg volume (ml/beat) & $<30$ & $30-44 ; 45-59$ & $\geq 60$ \\
\hline Reg fraction (\%) & $<30$ & $30-39 ; 40-49$ & $\geq 50$ \\
\hline $\operatorname{EROA}\left(\mathrm{cm}^{2}\right)$ & $<0.10$ & $0.10-0.19 ; 0.20-0.29$ & $\geq 0.30$ \\
\hline
\end{tabular}

AR, aortic regurgitation; EROA, effective regurgitant orifice area; LV, left ventricle; LVF, left ventricular function; LVOT, left ventricular outflow tract; PHT, pressure half time; reg, regurgitant.

well as artificial valve sizing. MDCT is often also used as an adjunct to trans-oesophageal echocardiography in assessing the aortic annulus and valve cusps. ${ }^{4,5}$ This fast-growing approach has intertwined the interventional cardiologist's and radiologist's expertise, as well as heralded the start of a dedicated 'heart team' for adequate assessment of the suitability of a patient prior to TAVI.

Several MDCT acquisition protocols, including dual energy virtual non-contrast (DEVNC) ${ }^{6}$ have been suggested and reviewed in quantifying the aortic valve and annulus prior to percutaneous intervention with TAVI. Some authors advocate the Agatston ${ }^{7,8}$ calcium scoring method, whilst others rely on a 'gestalt' or manual scoring system based on the Rosenhek diagrammatic calcium score ${ }^{9}$ in an attempt to predict post-procedural outcomes in these high-risk patients. Heinsig ${ }^{8}$ alluded to the fact that patients with a high Agatston aortic valve calcification burden were more prone to paravalvular leaks (PVL) as well as a subsequent increase in hospital morbidity and one-year mortality post TAVI. He demonstrated this within a study population of 120 patients. ${ }^{78}$ Although the Agatston aortic valve calcium score (AVCS) and its ability to predict the probability of a postprocedural paravalvular leak have been extensively written about in larger study groups, ${ }^{7,8,9}$ it has fallen out of favour at some institutions owing to its labour-intensive and timeconsuming reconstructions. Manual scoring systems, on the other hand, have failed to accurately predict complications in patients undergoing TAVI as they do not take into consideration the density of the calcified plaque.

The aim of the present study was to describe our local experience with MDCT in evaluating patients with clinically significant aortic stenosis, in preparation for TAVI, specifically proposing an alternative manual system for grading the calcification of the aortic valves annulus as well as the cusps, denoting calcification burden and density with single-source MDCT. We aimed to hypothesise a link between the Free State aortic valve calcium CT score (FACTS) and valve plaque averaged Hounsfield units (AHU), with patient/procedurerelated complications. We regarded complications such as paravalvular leak, stroke, death and myocardial infarction as major complications, and complications such as anaemia, left bundle branch block, and repositioning of the valve post/ during the procedure as minor complications.

\section{Research method and design Materials and setting}

A total of 20 patients from July 2011 - January 2013 were included. Consent was obtained from the hospital superintendent, prospective patients and the Faculty of Health Sciences Ethics Committee, University of the Free State, prior to the study (ECUFS no. 148/2012). Patients were evaluated by the Department of Cardiology, University of the Free State, and found eligible for TAVI. All fulfilled the criteria for clinically significant aortic stenosis, as determined by standardised inclusion criteria. ${ }^{10}$ No additional cardiac rhythm stabilising drugs were added to the patients' usual drug regime. Renal function was confirmed to be fit for intravenous contrast administration (Omnipaque 350, GE Healthcare Ireland, Cork, Ireland) prior to imaging. Exclusion criteria for imaging were severely impaired renal function $<40 \mathrm{ml} / \mathrm{min} / 1.73 \mathrm{~m}^{2}$ or previous severe adverse reaction to iodinated contrast material, such as anaphalactoid reaction, severe bronchospasm and cardiorespiratory collapse. ${ }^{11}$ Patients were evaluated by cardiac echocardiography (transoesophageal echocardiography (TEE) and trans-thoracic echo (TTE)) prior to and after TAVI at the cardiology interventional suite by dedicated echocardiographers using the Sellers technique as a guideline; see Table 1.

\section{Design}

This was a pro- and retrospective, double-blinded, cohort analytical study.

\section{Procedure}

Examinations were done on a single source, dual energy, high definition 64-detector CT scanner (HD750 Discovery, General Electric, Milwaukee). Patients were scanned using one of two standardised CT protocols, dependent on body weight ( $\geq 70 \mathrm{~kg}, 120 \mathrm{kV}, 160 \mathrm{ml} 350$ Omnipaque; and $\leq 70 \mathrm{~kg}$ $100 \mathrm{kV} 160 \mathrm{ml} 350$ Omnipaque), with ECG-prospectively gated, single-source energy scan of the chest, including gated cardiac CT and ungated single-source CTA of the abdomen and pelvis. Scan parameters were set with acquisition thickness of $0.625 \mathrm{~mm}$, small field of view of $25 \mathrm{~cm}$, a pitch of $0.16: 1$ and gantry rotation speed of $0.25 \mathrm{sec}$. $-0.35 \mathrm{sec}$, with $\mathrm{kV}$ and $\mathrm{mAs}$ dependent on body habitus. Scans were done in a craniocaudal direction on $75 \%$ of the RR interval. 


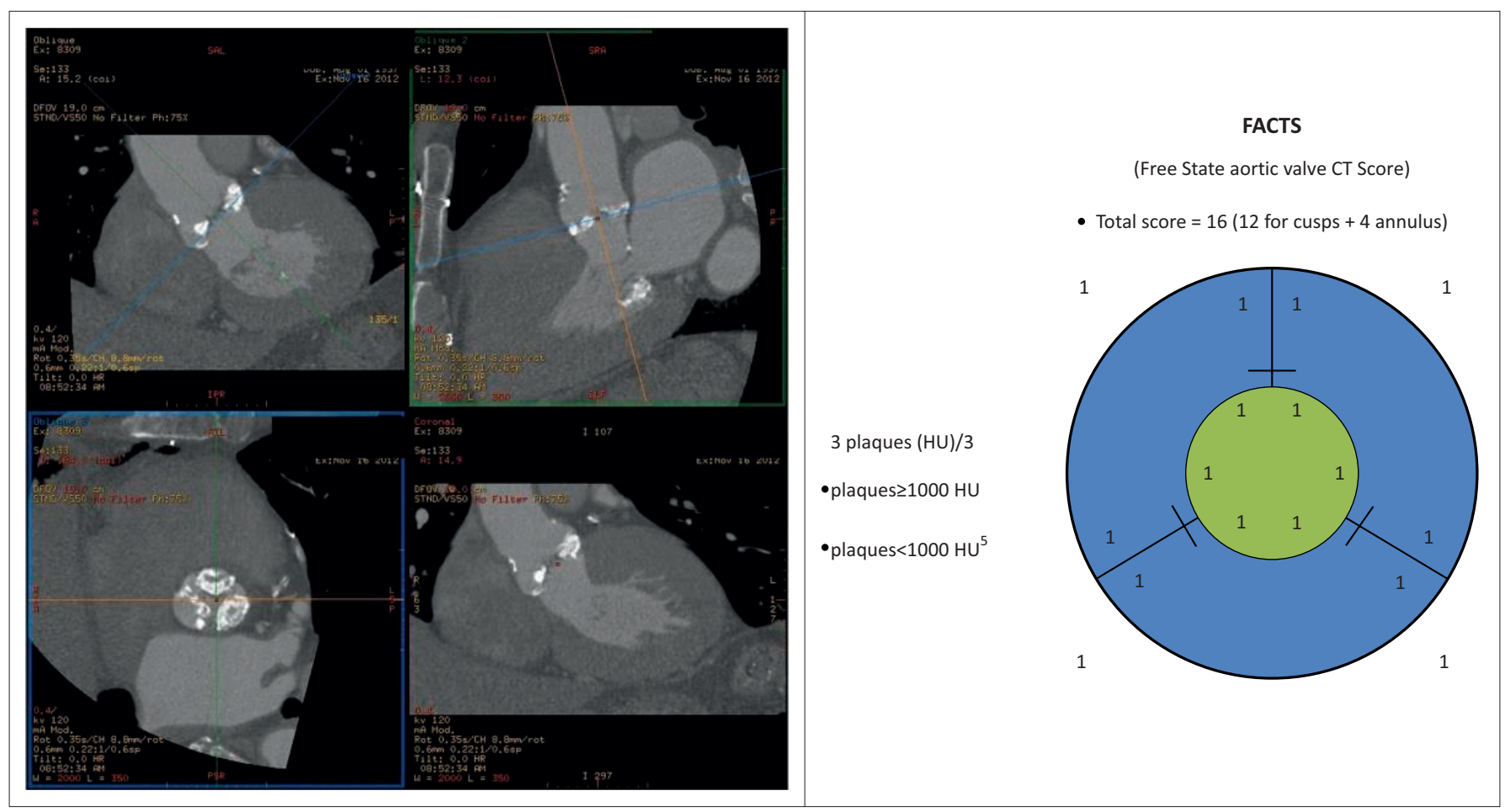

Source: Adapted from AVCS score where $>750 \mathrm{HU}$ had significant functional impairment,post procedural AR, increased 1-year mortality and procedural complications

Leber AW, Kasel M, Ischiger T, et al. Aortic valve calcium score as a predictor for outcome afterTAVI using the Core Valve revalving system. Int J Cardiol. 2011. http://dx.doi.org/10.1016/j.ijcard.2011/ 11/091FACTS

FIGURE 1: Schematic representation of the FACTS score average Hounsfield units (HU) score. Each number represents $25 \% / 1$ point of the valve cusp $=$ total score of 12 . The outer numbers represent $25 \% / 1$ point of the annulus. Total score of both aortic annulus and cusps therefore $=16$. The most heavily calcified plaque on each cusp was measured in $\mathrm{HU}$ and divided by a factor of 3 to obtain an average score of either $\geq 1000 \mathrm{HU}$ or $<1000 \mathrm{HU}$ ). Annulus calcifications were not taken into account with the HU scores.

Automated bolus injection was implemented with the region of interest placed over the ascending aorta with scanning of the chest commencing at an attenuation value of $120 \mathrm{HU}$. Contrast was administered via an automated pump at $5 \mathrm{ml} /$ sec., with a $40 \mathrm{ml}$ saline bolus 'chaser' given after contrast injection.

\section{Imaging analysis}

Images were reviewed weekly by a qualified radiologist and a training fellow at the University of the Free State, Universitas Hospital Complex. Post processing of images took place on an Advantage Workstation 4.5 (General Electric) and analyses were performed using standard software (Reformat, General Electric). Data were loaded into a standard multiplanar reformat package, with image reconstructions in the coronal, sagittal and axial orientation and analysed using a multiplanar oblique tool. The coronal image was used to visualise the left ventricular outflow tract, with placement of the oblique tool in the middle of the ascending aorta, producing a sagittal oblique view. Placement of the oblique tool was in the centre axis of the ascending aorta, producing true axial images in the aortic leaflets and annulus planes, on which the calcification scoring was done. The aortic annulus and valve leaflets calcification burden was scored using a bone window level (WW2000 WL350).

Two views were used with the FACTS scoring of the aortic valve. The first view visualised the entire aortic annulus on the modified true axial views as done by using the multiplanar oblique tool. Outer ring scoring was done on this initial view. The second view visualised the distal ends of the leaflets as they converged; inner ring scoring was done on this view. The aortic annulus was divided into quarters on the first view and scored. One point was given for every calcification/plaque seen on the individual regions as described. The total score added to 16 , where each valve had 4 possible sections as well as the annulus, which was also divided into 4 sections (Figure 1).

\section{FACTS, Rosenhek and HU Score}

FACTS scores were done individually and each observer blinded to the other's score. These scores were correlated to the Rosenhek diagrammatic calcium valve scoring system (Figure 2). The diagrammatic Rosenhek scoring system is shown in Figure 3.

Patients for whom the observers' FACTS scores showed a difference $>15 \% / 2$ points were rescored, after retraining, by the same two observers and included in the final data set for statistical analysis. An agreement score was seen as a difference of up to $10 \%-15 \% / 2$ points between observers. Complications were associated with scores where the two observers were in agreement after the final scoring. Eighteen patients' FACTS scores and 16 patients' Rosenhek scores matched in the final analysis.

HUs were done separately to the FACTS scores and calculated on each aortic valve cusp. The largest calcified plaque density 


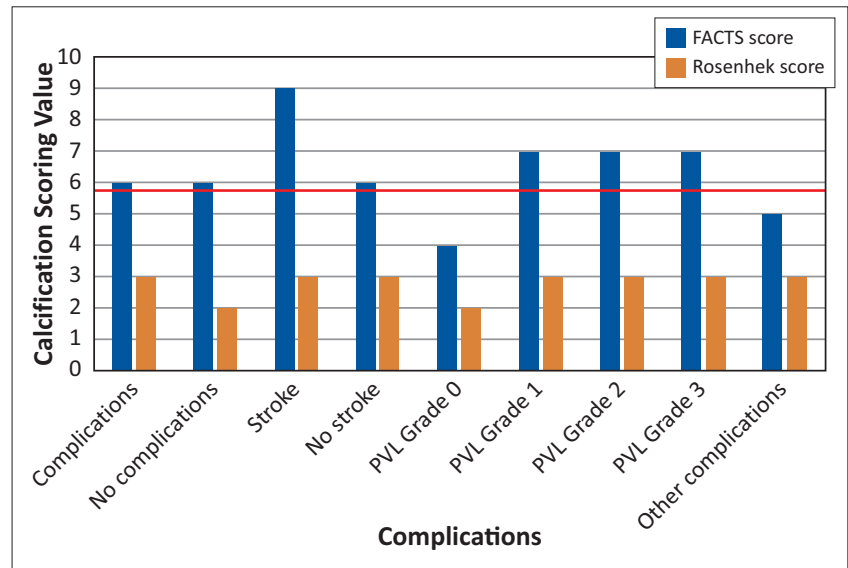

PVL, paravalvular leak. Other complications = left bundle branch block, anaemia, atrial fibrillation.

FIGURE 2: FACTS and Rosenhek scores v. complications.

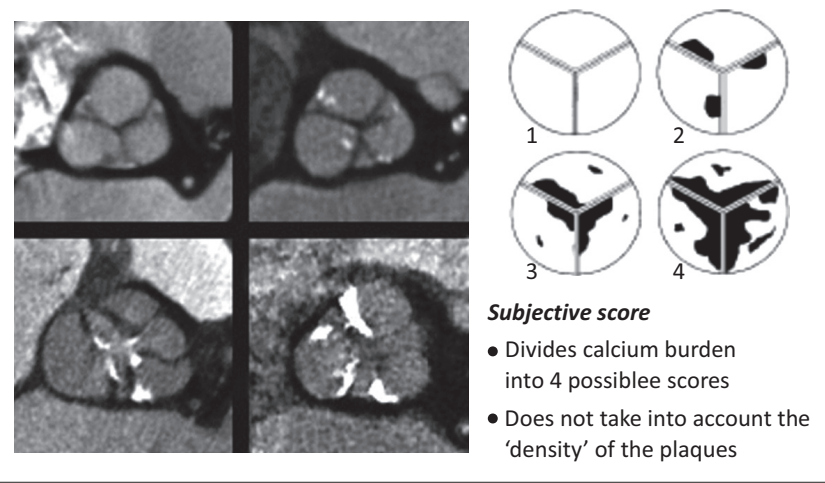

Source: Adapted from Willman JK, Weishaupt D, Lachat M, et al. Electrocardiographically gated multi-detector row CT for asessment of valvular morphology and calcification in aortic stenosis. Radiology. 2002;225:120-128.

FIGURE 3: Rosenhek score.

was taken on each valve cusp and summed. This figure was divided by a factor of 3 for an average score and grouped to either $\geq 1000 \mathrm{HU}$ or $<1000 \mathrm{HU}$ as agreed upon between the observers, taking an $\mathrm{HU} \geq 1000$ as denoting calcified bone on routine CT. Correlations between the two observers' AHU scores were measured and 18 scores matched according to category.

All data (original and rescored) were captured and typed into a Microsoft Excel spreadsheet for statistical analysis. Age, race, sex, comorbid factors, grades of dyspnoea, left ventricular outflow fraction (prior and after the procedure) and presence of aortic regurgitation, complications and the presence of a pacemaker prior to and after procedures were included. Complications considered as major were stroke, myocardial infarction, death, paravalvular leak and 'other'. 'Other' included minor complications such as repositioning of the valve, anaemia post procedure and left bundle branch block. Patients' were followed up at 30,90 and 180 days. No patients died during the follow-up period.

\section{Results}

A total of 20 patients ( 9 men [45\%] and 11 women [55\%]), who happened to all be white, were enrolled in the study. Only
TABLE 2: Patient demographics.

\begin{tabular}{lll}
\hline Patient demographics & \multicolumn{2}{c}{ Age (median and range) } \\
\cline { 2 - 3 } & $\mathbf{8 3 . 5}$ years & $\mathbf{6 8}$ years-95 years \\
\hline Gender & $45 \%$ male and 55\% female & - \\
EuroSCORE (median and range) & 15.5 & $5-22$ \\
\hline NYHA & Prior to TAVI & After TAVI \\
Average & 3 & 2 \\
LVEF & Prior to TAVI (TEE) & After TAVI (TEE) \\
& $55 \%$ & $61.5 \%$ \\
Pacemakers & Prior & After \\
& 1 & 3 \\
Aortic regurgitation & Prior to TAVI & PVL post TAVI \\
Grade 0 & $20 \%$ & $20 \%$ \\
Grade 1 & $80 \%$ & $50 \%$ \\
Grade 2 & - & $25 \%$ \\
Grade 3 & - & $5 \%$ \\
\hline
\end{tabular}

NYHA, New York Heart Association; LVEF, left ventricular ejection fraction; TAVI, trans-catheter aortic valve implantation; TEE, trans-oesophageal echocardiography; PVL, paravalvular leak.

18 patients' FACTS scores were used in the final analysis. Patient demographics are shown in Table 2.

New York Heart association (NYHA) criteria were recorded prior to and following the procedure, with $35 \%$ of patients being NYHA 2, 50\% NYHA 3, and 15\% NYHA 4 prior to the procedure and showed marginal improvement post TAVI (NYHA 1 (10\%), NYHA 2 (70\%), and NYHA 3 (20\%), but this was not statistically significant $(p=0.41)$. The median age of the patients was 83.5 years (range 68-95 years), with a EuroSCORE median of 15.5 (range: $5-22$ ).

Sixteen $(80 \%)$ of the 20 patients had a grade 1 aortic incompetence prior to TAVI, and 17 (85\%) had paravalvular leak (PVL) post TAVI, of whom 10 patients had grade 1 PVL, 5 patients had grade 2 PVL, and 1 patient had grade 3 PVL. FACTS scores $\geq 6$ were significantly associated with the presence of a paravalvular leak $(p=0.01)$ post TAVI. Grade 1 PVL $=$ mild PVL, Grade 2 PVL $=$ moderate PVL, and Grade $3 \mathrm{PVL}=$ severe PVL. The FACTS scores were similar to the Rosenhek score in this regard, and both major and 'other' complications were seen to arise at a Rosenhek score $>2$, but were not statistically significant $(p=1.00$; 'other' complications $p=0.09$ ). No patient suffered from a myocardial infarction or death during or immediately after the procedure (see Figure 2). Nine patients fell into the 'other' complication category. The totals of complications were similar in both Rosenhek score groups, with both groups having close to the same number of complications in the 'other' category.

The median left ventricular ejection fraction (LVEF) was $56.5 \%$ (range $27 \%-77 \%$ ) and marginally increased to $61.5 \%$ (range 29\%-75\%) at follow-up. Comorbid conditions (see Figure 4) prior to TAVI were recorded and were as follows: hypertension $-80 \%$, diabetes $-15 \%$, renal impairment $-10 \%$ ), ischaemic heart disease $-35 \%$ ), hypercholesterolaemia $-24 \%$, chronic lung disease $-65 \%$, peripheral vascular disease $65 \%$, and congestive cardiac failure $-5 \%$.

Four patients received pacemakers; 1 was inserted prior to TAVI and 3 post TAVI. Left bundle branch block is a known complication of the TAVI procedure. All of our patients had 


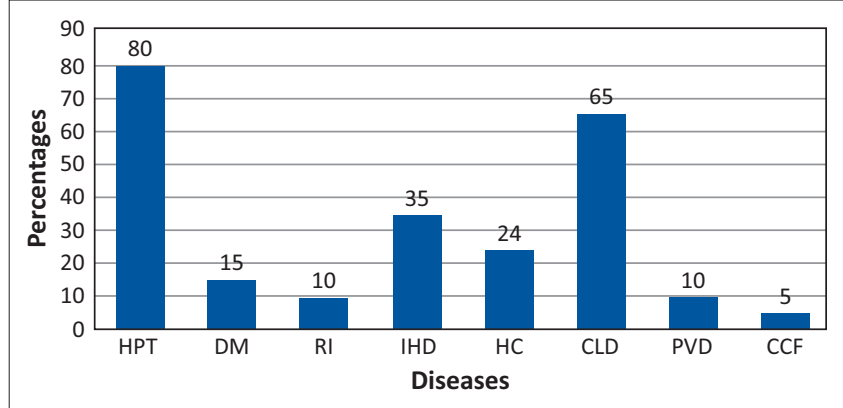

HPT, hypertension; DM, diabetes; RI, renal impairment; IHD, ischaemic heart disease; HC, high cholesterol; CLD, chronic lung disease; PVD, peripheral vascular disease; CCF, congestive cardiac failure.

FIGURE 4: Comorbid factors prior to TAVI.

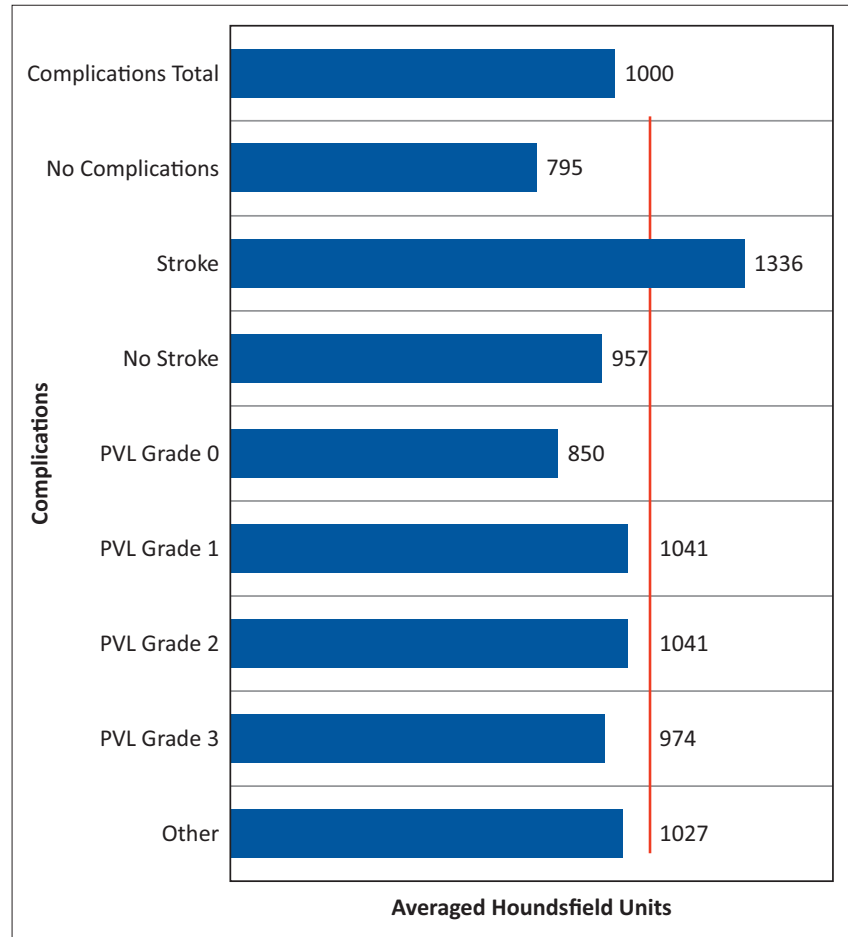

PVL, paravalvular leak.

FIGURE 5: Averaged Hounsfield units versus complications.

degenerative aortic stenosis. An AHU $\geq 1000$ was associated with 'other complications' amongst which left bundle branch block, anaemia post procedure and repositioning of the valve were seen; this was close to being statistically significant $(p=0.07)$. A graph of AHU v. complications is shown in Figure 5. AHUs ranged from 795 to 1336.

Only one female patient had a transient ischaemic event, which resolved in 24 hours. She had high FACTS and AHU scores (FACTS $=9$ and AHU $\geq 1000$ ). Male patients also had a higher incidence and grade of PVL than female patients, which we associated with a plaque density $\geq 1000 \mathrm{HU}$. One patient had a grade 3 PVL that was the result of initial incorrect positioning of the artificial valve, with a grade 1 PVL after repositioning of the Medtronic valve.

Plaques $\geq 1000 \mathrm{HU}$ were associated with comorbidities; diabetes was found to be the main comorbid factor in plaque density differences, as seen in Figure 6.

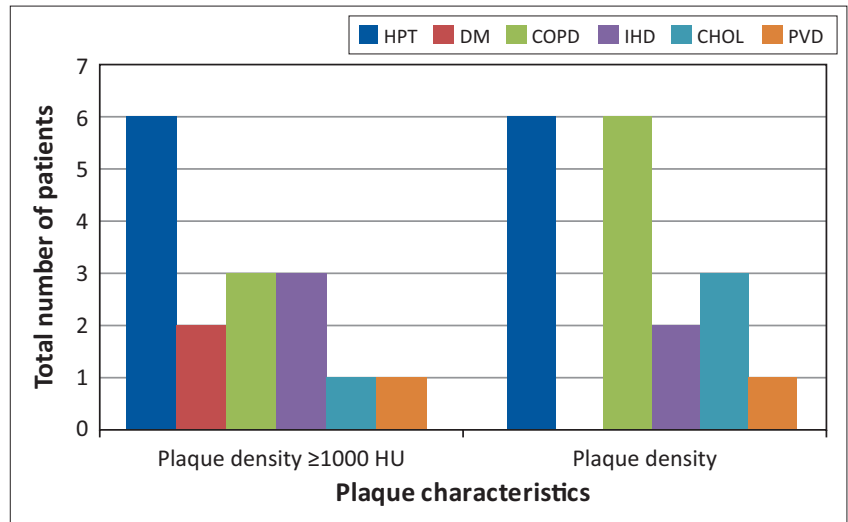

HPT, hypertension; DM, diabetes; RI, renal impairment; IHD, ischaemic heart disease; HC, high cholesterol; CLD, chronic lung disease; PVD, peripheral vascular disease; CCF, congestive cardiac failure.

FIGURE 6: Plaque characteristics v. comorbidities.

No adverse events were recorded either during or after image acquisition. All TAVI procedures were performed after multidisciplinary discussion, using a transfemoral approach by a dedicated cardiac team, using the self-expandable Medtronic CoreValve.

\section{Statistical analysis}

Results were summarised by frequencies and percentages (categorical variables) and means or percentiles (numerical variables, depending on the distribution of the data). Groups were compared using chi-squared, Fisher's exact test or McNemar's test for categorical variables, the Kruskal-Wallis test, or Mann-Whitney test for numerical variables.

\section{Ethical considerations}

Benefits and hazards: If an association between plaque burden and density could be drawn to FACTS score $\geq 6$ and $H U \geq 1000$, pre-procedural evaluation could be streamlined and post-procedural complications be anticipated and timeously treated.

Recruitment procedures: Patients undergoing preinterventional imaging were asked to take part in the study. Prospective and retrospective data capturing was done. No monetary compensation was offered to patients for taking part in the study. There was no discrimination against patients who declined participation.

Informed consent: Each patient received an information document, explaining the goal of the study and the imaging procedure involved. Patients were asked to sign this document prior to imaging, if they agreed to participate in the study.

Data protection: Patients' data were captured onto a standardised Excel sheet and numbers allocated to each patient. Only the first observer/researcher had access to patients' names. Confidentiality was maintained in all circumstances. 


\section{Trustworthiness}

All TAVI procedures were performed by a dedicated cardiology team with the same two operators, with the standard use of a transfemoral approach. All patients' aortic valves were reviewed, and calcified valve plaques scored by the same two radiology reviewers on the same GE workstation, using a standard double-blinded method.

\section{Discussion}

TAVI is an established percutaneous method for the

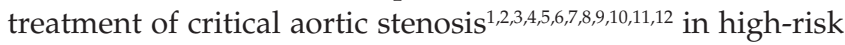
patients and is relatively new in the South African context. ${ }^{12}$ The Agatston calcification scoring system ${ }^{5,7,8,10}$ for aortic valve calcification has been proposed in the past as well as manual valve plaque calcification scoring systems ${ }^{9}$ with and without the use of computer software, linking complications such as paravalvular leak and long-term patient survival to these scores ${ }^{13,14,15}$ and proposing better patient selection prior to intervention.

We aimed to introduce an alternative manual scoring system that was easy to implement, took plaque density into account, was not time consuming, and the same time could predict clinically significant adverse patient outcomes. In our small study sample, we found that a FACTS score $\geq 6$ was significantly associated with the presence of a paravalvular leak. Literature has shown that paravalvular leak post TAVI is an independent risk factor for longer hospital stay as well as an increased mortality risk factor at one-year follow-up. ${ }^{7}$ Procedure-related complication was close to statistically significantly associated with average valve plaque densities $\geq 1000$ HU. Unfortunately, we were not able to differentiate between grades of paravalvular leak in our study, which could be attributed to our attempt to introduce a new manual scoring system and/or that our study population was small. Nevertheless, all the artificial valves were inserted by the same dedicated cardiology team, so the fact that an association between AHU score and complications such as anaemia, left bundle branch block and repositioning of the artificial valve could be shown is, we feel, some achievement.

We used the Rosenhek score as a reference standard, although in hindsight the two scoring systems are inherently different in that the FACTS and AHU scores offer quantification as well as qualification of the calcified plaques versus the firstmentioned score that only relies on a 'gestalt' or quantification of the calcification burden of the aortic valves, excluding the aortic annulus. Our score included the aortic annulus which, we postulate, could play a role in the pathology of paravalvular leaks post TAVI in our population. A possible reason for this could be the limited pliability of the aortic annulus with bulky calcified plaque. The Rosenhek score could also not differentiate between specific complications (e.g. stroke v. PVL) in our small study population but was, however, associated with no complications if the score was $<2$, when compared with the FACTS score; and, interestingly, the Rosenhek scores were seen to match in $80 \%$ of cases between the two observers, in the final statistical analysis.
The LVEF and NYHA only marginally improved after TAVI procedure and were found to be statistically unrelated to either the FACTS or AHU scores. Pacemaker insertion could also be linked to high AHU scores, although this was not statistically significant and is a known complication of the Medtronic CoreValve. ${ }^{13}$

\section{Limitations}

In our study population, complications were minimal, which could be due to the small study size or because the TAVIs were done meticulously by a qualified cardiac team. Moderate inter-individual variations were seen with the FACTS scores initially but, after retraining, the differences were markedly less. One explanation for the variation could be the relative inexperience of both observers with a new scoring system or because of the lack of experience in calcification scoring. Only two observers scored in this article. Maybe a larger number of observers could have improved the results. The FACTS and AHU scores were looked at individually and could have been incorporated into a single scoring system.

\section{Recommendation}

We recommend the incorporation of the AHU with the FACTS score in future for a uniform score, giving a specific numeral value to a specific HU density as well as a larger sample size. Individual scoring of the annulus versus the cusp calcification could also be considered for future studies.

\section{Conclusion}

The FACTS and AHU scores are easy to implement, not labour intensive or time consuming, and show promise in providing important added information for patients undergoing pre-interventional assessment for the TAVI procedure, especially in determining the risk of paravalvular leak, left bundle branch block, repositioning of the artificial aortic valve and anaemia. A larger study sample is necessary to confirm these preliminary findings.

\section{Acknowledgements}

The authors thank Dr W. Harmse, diagnostic radiologist and current fellow in Interventional Radiology, Alberta Health Services, Foothills Medical Centre; Professor S. Brown, Department of Paediatric Cardiology, University of the Free State; Professor H. Theron, Head of Department Cardiology, Universitas Academic Hospital, University of the Free State; and Mrs C. Swanepoel, Chief Radiographer Computed Tomography, Department of Clinical Imaging Sciences, University of the Free State, for their invaluable contribution to the completion and publication of the study.

\section{Competing interests}

The authors declare that they have no financial or personal relationships which may have inappropriately influenced them in writing this article. 


\section{Authors' contributions}

A.R. (University of the Free State) protocol and study design, first blinded observer, writing and planning of the article draft and final version, main corresponding author. C.S.d.V. (University of the Free State) second blinded observer, study coordinator, protocol and final article draft reviewer. Professor G.J. (University of the Free State) is thanked for reviewing the statistics and protocols and for her invaluable input in the final draft of this article.

\section{References}

1. Webb J, Rodes-Cabau J, Fremes $\mathrm{S}$, et al. Transcatheter aortic valve implantation: A Canadian Cardiovascular Society position statement. Can J Cardiol. 2012;28: 520-528. http://dx.doi.org/10.1016/j.cjca.2012.04.015

2. Anderson HR, Knudsen LL, Hasenkam JM. Transluminal implantation of artificial heart valves: Description of new expandable aortic valve and initial results with implantation by catheter technique in closed chest pigs. Eur Heart J. 1992;13: 704-708.

3. Cribier A, Eltchaninoff $H$, Bash $A$, et al. Percutaneous transcatheter implantation of an aortic valve prosthesis for calcific aortic stenosis. Circulation. 2002;106: 3006-3008. http://dx.doi.org/10.1161/01.CIR.0000047200.36165.B8

4. Tamborini G, Fusini L, Gripari P, et al. Feasibility and accuracy of 3DTEE versus $\mathrm{CT}$ for the evaluation of aorticvalve annulus to left main ostium distance before transcatheter aortic valve implantation. JACC Cardiovasc Imaging. 2012;5: 579-588. http://dx.doi.org/10.1016/j.jcmg.2012.02.012

5. Rixe J, Schuhbaeck A, Liebetrau C, et al. Multi-detector computed tomography is equivalent to trans-oesophagal echocardiography for the assessment of the aortic
annulus before transcatheter aortic valve implantation. Eur Radiol. 2012;22: annulus before transcatheter aortic valve implantation.
2662-2669. http://dx.doi.org/10.1007/s00330-012-2530-2
6. Mahoney R, Pavitt CW, Gordon D, et al. Clinical validation of dual-source dualenergy computed tomography for coronary and valve imaging in patients undergoing trans-catheter aortic valve implantation. Clin Radiol. 2014;69: 786-794. http://dx.doi.org/10.1016/j.crad.2014.03.010

7. Haensig $\mathrm{M}$, Lehmkuhl L, Rastan AJ, et al. Aortic valve calcium scoring is a predictor of significant paravalvular aortic insufficiency in transapical-aortic valve implantation. Eur J Cardiothorac Surg. 2012;41:1234-1241. http://dx.doi.org/ 10.1093/ejcts/ezr244

8. Haensig M, Rastan JA. Aortic valve calcium load before TAVI: Is it important? Ann Cardiothorac Surg. 2012;1:160-164. http://dx.doi.org/10.3978/j.issn.2225319X.2012.06.02

9. Willman JK, Weishaupt D, Lachat $M$, et al. Electrocardiographically gated multi-detector row CT for asessment of valvular morphology and calcification in aortic stenosis. Radiology. 2002;225:120-128. http://dx.doi.org/10.1148/ radiol.2251011703

10. Leipsic J, Gurvitch R, LaBounty TM, et al. Multidetector computed tomography in transcatheter aortic valve implanation. JACC. 2011;4:416-419. http://dx.doi. org/10.1016/j.jcmg.2011.01.014

11. Blanke $P$, Euringer $W$, Baumann $T$, et al. Combined assessment of aortic root anatomy and aortoiliac vasculature with dual-source CT as a screening tool in patients evaluated for transcatheter aortic valve replacement. Am J Roentgenol. 2010;195:872-881. http://dx.doi.org/10.2214/AJR.10.4232

12. Weich $\mathrm{H}$, Mabin T, Van Wyk J, et al. First experience with the Edward SAPIEN transcatheter aortic valve implantation (TAVI). Data from the Western Cape, South Africa. SA Heart J. 2012;9:6-13.

13. Leber AW, Kasel $M$, Ischinger $T$, et al. Aortic valve calcium score as a predictor for outcome after TAVI using the Corevalve revalving system. Int $\mathrm{J}$ Cardiol. 2011;166:652-657. http://dx.doi.org/10.1016/j.ijcard.2011.11.09

14. Feuchtner GM, Muller S, Grander $W$, et al. Aortic valve calcification as quantified with multislice computed tomography predicts short-term clinical outcome in patients with asymptomatic aortic stenosis. J Heart Valve Dis. 2006;15:494-498.

15. Delgado $\mathrm{V}, \mathrm{Ng} \mathrm{ACT}$, van de Veire $\mathrm{N}$, et al. Transcatheter aortic valve implantation: Role of multidetector row computed tomography to evaluate prosthesis positioning and deployment in relation to valve function. Eur Heart J. 2010;31:1114-1123. $\mathrm{http}: / /$ dx.doi.org/10.1093/eurheartj/ehq018 